\title{
Studies towards the total synthesis of novel dolabellane-type diterpenoids: construction of the 5,11-fused bicyclic framework
}

\author{
Goverdhan Mehta* and Jayant D. Umarye \\ Department of Organic Chemistry, Indian Institute of Science, Bangalore 560 012, India
}

\begin{abstract}
An oxy-Cope protocol has been grafted on to a previously crafted hydroazulenic system to provide a facile entry into the 5,11-fused bicyclic skeleton present in the novel neodolabellane diterpenoids. Formation of a novel tricyclic diterpenoid framework, generated through a transannular cyclization in the 11-membered ring present in the neodolabellane skeleton and embodying a bridged cycloheptatriene moiety, has also been encountered.
\end{abstract}

Dolabellane diterpenes bearing a novel 5,11-fused bicyclic framework 1 were for the first time encountered way back in 1976 from the digestive glands of the sea hare Dolabella California sterns ${ }^{1 \mathrm{a}}$ and since then natural products based on this skeleton have surfaced from marine and terrestrial sources at regular intervals, constituting one of the largest group among diterpenoids. ${ }^{1}$ Sharing close biogenetic relationship with dolabellanes are the 5,7,6-fused tricyclic diterpenoids of dolastanetype 2 (isoamijiol) ${ }^{2 \mathrm{c}}$ which are also widely occurring in Nature. ${ }^{2}$ Both dolabellane and dolastane diterpenoids have aroused a great deal of synthetic interest both on account of the novelty of their carbocyclic skeleta and the dense oxy-functionalization present in them. . $^{3,4}$
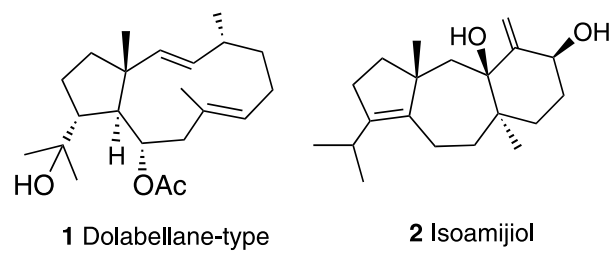

2 Isoamijiol

Subsequently, the neodolabellane skeleton represented by $3, \alpha$ - and $\beta$-neodolabellenol, ${ }^{\text {sc }}$ and biogenetically derived from a dolabellane precursor through stereospecific methyl migration was also reported from marine organisms. ${ }^{5}$ More interestingly, a 5,7,6-fused tricyclic diterpeneoid guanacastepene A 4, having the same biogenetic kinship with neodolabellanes, as dolastanes $\mathbf{2}$ have with dolabellane 1, has been very recently

\footnotetext{
* Corresponding author.
}

reported ${ }^{6}$ from an unidentified endophytic fungus growing on the tree Daphnopsis Americana and exhibiting remarkable antibiotic activity. Both, neodolabellane and guanacastepene diterpenoids constitute challenging targets for synthesis. While several groups have been in pursuit of guanacastepenes, ${ }^{7}$ the group of Williams ${ }^{8}$ is the only one to report the synthetic accomplishment of neodolabellane natural products. In view of our ongoing interest in the synthesis of diterpenoids $\mathbf{1}-\mathbf{4}^{30,4 \mathrm{j}, 7 \mathrm{a}-\mathrm{c}}$ we have devised an entry into the highly functionalized 5,11-fused bicyclic system present in neodolabellanes in which an oxy-Cope rearrangement is the pivotal step and these and related studies are reported in this letter.

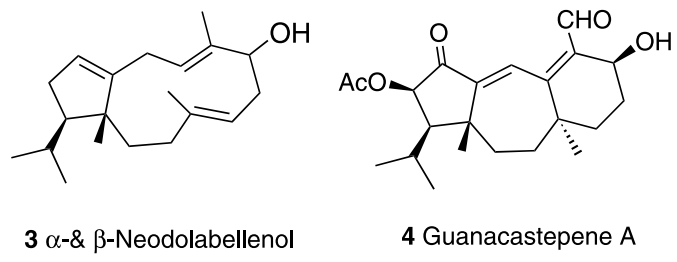

We have recently described the synthesis of hydroazulenone 5 from dicyclopentadienone $\mathbf{6}$ in the context of our approach to the synthesis of guanacastepene A 4. ${ }^{7 \mathrm{a}-\mathrm{c}}$ The seven membered ring of hydroazulenone $\mathbf{5}$, having the angular methyl and the isopropyl groups in correct cis-stereochemical disposition, appeared well poised for the execution of a four carbon ring enlargement protocol to deliver the desired 5,11-fused bicyclic ring system of neodolabellanes. Our first foray in this context involved a four carbon ring annulation followed by a Grob-type fragmentation. Consequently, 
hydroazulenone 5 was elaborated to the tricyclic system 9 through a sequence involving $\alpha$-carboethoxylation and stereoselective allylation to 7 and further addition of the Grignard reagent derived from 3-chloro-1-butene led to 8 as described previously (Scheme 1). ${ }^{7 \mathrm{c}} \mathrm{RCM}$ reaction in 8 delivered the tricyclic product 9 . LAH reduction of 9 furnished the tricyclic diol 10a to set-up the intended fragmentation reaction. Exposure of 10a to methanesulfonyl chloride in the presence of base led to the isolation of a single product which was assigned the novel bridged structure $\mathbf{1 3}$ on the basis of incisive ${ }^{1} \mathrm{H}(\mathrm{COSY})$ and ${ }^{13} \mathrm{C}$ NMR studies. ${ }^{9}$ Formation of $\mathbf{1 3}$ indicated that the Grob-type fragmentation in 10a had indeed occurred through the mesylate 10b to form the expected bicyclic neodolabellane-type product $\mathbf{1 1}$ (Scheme 1). However, in the reaction medium, 11 undergoes an extremely facile transannular carbonylene cyclization to $\mathbf{1 2}$ through the participation of the exocyclic methylene group and further dehydration results in the observed product $\mathbf{1 3}$ (Scheme 1). It may be recalled that bridged cycloheptatriene derivatives similar to $\mathbf{1 3}$ have been encountered among sesquiterpenoid natural products, ${ }^{10}$ but no diterpenoid framework with this novel structural feature is known. Many variations of the Grob-type fragmentation in both 9 and 10a were explored but it was not possible to isolate any 5,11-fused bicyclic product. Recourse was therefore taken to effect an oxy-Cope rearrangement ${ }^{11}$ in a suitably crafted derivative of $\mathbf{5}$ to effect a four-carbon ring expansion.

To set up the precursor for the oxy-Cope process, hydroazulenone $\mathbf{5}$ was elaborated through sequential $\alpha$-carboethoxylation and methylation to furnish $\mathbf{1 4}$ as a single diastereomer. Addition of the Grignard reagent prepared from 2-bromopropene to $\mathbf{1 4}$ was also stereoselective and furnished $\mathbf{1 5}$ (Scheme 2). The ester functionality in $\mathbf{1 5}$ was converted to the aldehyde $\mathbf{1 6}$ in two steps involving LAH reduction to the primary alcohol and reoxidation employing IBX. Wittig olefination of the aldehyde 16 afforded the divinyl carbinol 17, the precursor for the oxy-Cope rearrangement (Scheme 2). Attempted, anionic oxy-Cope rearrangement on 17 employing $\mathrm{KH}$ or KHMDS or $\mathrm{NaH}$ as the base, with or without phase transfer catalysis and under different solvent regimes (THF, DME, dioxane) was singularly unsuccessful. However, thermal activation of $\mathbf{1 7}$ in boiling 1,2-dichlorobenzene induced the [3.3]-sigmatropic shift and oxy-Cope rearrangement products $18 / 19$ were obtained as a mixture (1:1) of methyl epimers in modest yield (Scheme 2). While 18 and 19 could be separated and fully characterized, it was not possible to assign unambiguously stereochemistry to the newly generated secondary methyl group in either case despite many 2D NMR experiments. To demonstrate the generality of this oxy-Cope process, aldehyde $\mathbf{1 6}$ was subjected to a Horner-Wittig reaction to afford the $(E)-\alpha, \beta$-unsaturated ester $\mathbf{2 0}$ as a single diastereomer. Thermal activation of 20, under conditions identical to those employed for 17, furnished the [3.3]-shift product 21, quite fortuitously as a single diastereomer and in much better yield (Scheme 3). While the gross structure of 21 was readily forthcoming from ${ }^{1} \mathrm{H}$ and ${ }^{13} \mathrm{C}$ NMR data, recourse to even high field 2D NMR (COSY, NOESY) studies was inadequate to deduce the stereostructure of this oxy-Cope rearrangement product.
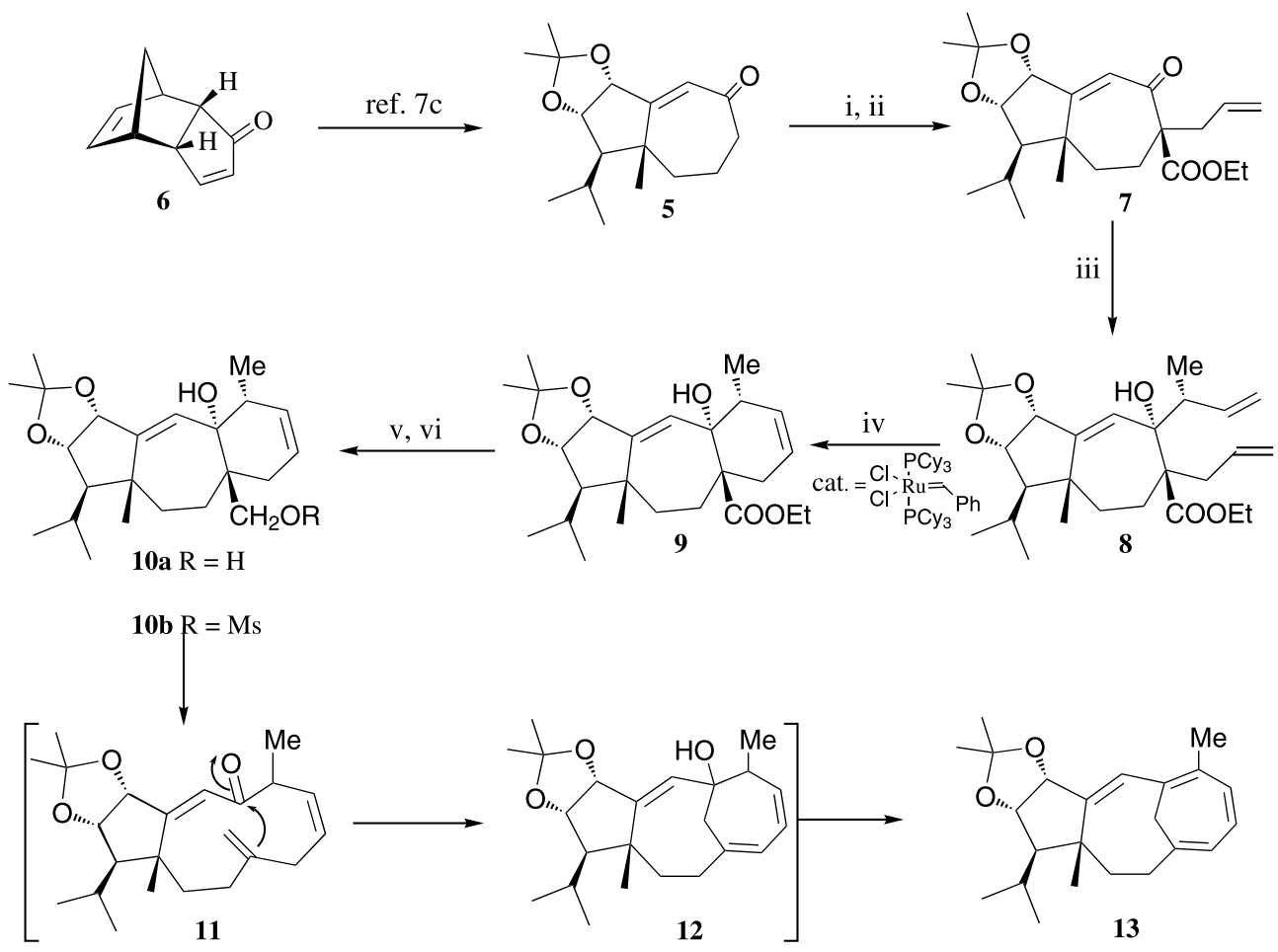

Scheme 1. Reagents and conditions: (i) LDA, THF-HMPA, CNCOOEt, $-78^{\circ} \mathrm{C}, 75 \%$; (ii) NaH, THF, allyl bromide, rt, $95 \%$; (iii) $\mathrm{Mg}$, 3-chloro-1-butene, $\mathrm{CeCl}_{3}$, THF, 60\%; (iv) cat. (10 mol\%), benzene, $\Delta, 96 \%$; (v) LAH, THF, $\Delta, 65-70 \%$; (vi) $\mathrm{NEt}$, $\mathrm{MsCl}$, DCM, 70\%. 

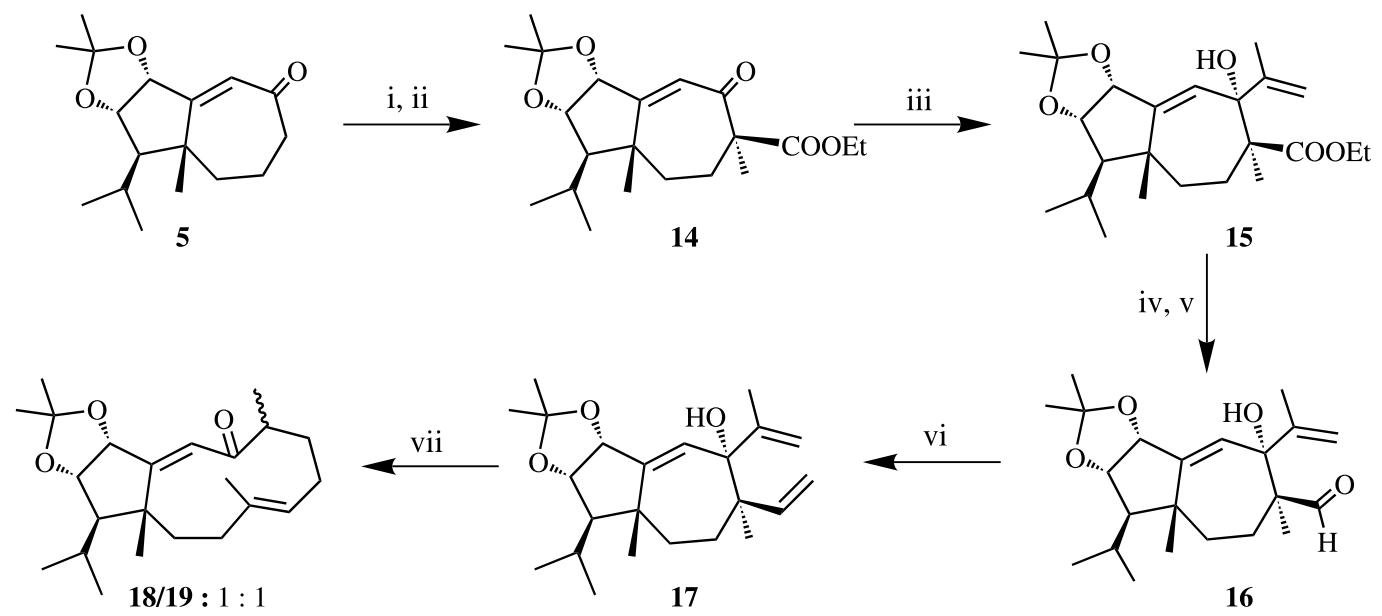

Scheme 2. Reagents and conditions: (i) LDA, THF-HMPA, CNCOOEt, $-78^{\circ} \mathrm{C}, 75 \%$; (ii) $\mathrm{NaH}, \mathrm{THF}, \mathrm{MeI}, \mathrm{rt}, 95 \%$; (iii) $\mathrm{Mg}$, 2-bromopropene, THF, $0^{\circ} \mathrm{C}-\mathrm{rt}, 80-85 \%$; (iv) $\mathrm{LAH}$, THF, rt, 98\%; (v) IBX, toluene/DMSO (4:1), $85 \%$; (vi) $\mathrm{MePh}_{3} \mathrm{P}^{+} \mathrm{Br}^{-}, \mathrm{KO}^{t} \mathrm{Bu}$, THF, quant.; (vii) $o$-DCB, $180^{\circ} \mathrm{C}, 10 \mathrm{~h}, 35 \%$.

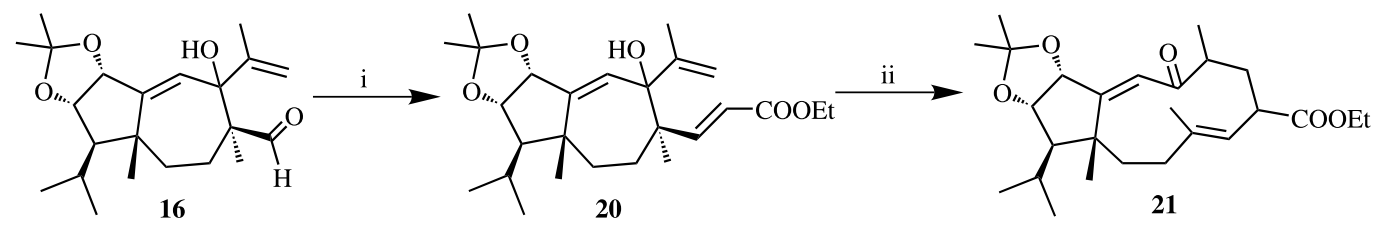

Scheme 3. Reagents and conditions: (i) $(\mathrm{EtO})_{2} \mathrm{P}(\mathrm{O}) \mathrm{CH}_{2} \mathrm{COOEt}, \mathrm{NaH}, \mathrm{THF}, \mathrm{rt}, 2 \mathrm{~h}, 90 \%$; (ii) $o-\mathrm{DCB}, 180^{\circ} \mathrm{C}, 6 \mathrm{~h}, 60 \%$.

In summary, we have devised an oxy-Cope rearrangement based strategy to access the 5,11-fused bicyclic framework of novel neodolabellane diterpenes, replete with requisite stereochemical features at two key stereogenic centers and extensive functionalization, from a readily accessible hydroazulenic precursor.

\section{Acknowledgements}

J.D.U. thanks CSIR for the award of a research fellowship. We also acknowledge the help from SIF at the IISc towards obtaining high field NMR spectral data. This research was supported by the Chemical Biology Unit of JNCASR in Bangalore.

\section{References}

1. (a) Ireland, C.; Faulkner, D. J.; Finer, J.; Clardy, J. J. Am. Chem. Soc. 1976, 98, 4664; (b) Ireland, C.; Faulkner, D. J. J. Org. Chem. 1977, 42, 3157; (c) Sun, H. H.; Fenical, W. Phytochemistry 1979, 18, 340; (d) Amico, V.; Oriente, G.; Piattelli, M.; Tringali, C.; Fattorusso, E.; Mango, S.; Mayol, L. Tetrahedron 1980, 36, 1409; (e) Tringali, C.; Piattelli, M.; Nicolosi, G. Tetrahedron 1984, 40, 799; (f) Rodriguez, A. D.; Gonzalez, E.; Ramirez, C. Tetrahedron 1998, 54, 11683; (g) Asakawa, Y.; Lin, X.; Tori, M.; Kondo, K. Phytochemistry 1990, 29, 2597.

2. (a) Pettit, G. R.; Ode, R. H.; Herald, C. L.; Von Dreele, R. B.; Michel, C. J. Am. Chem. Soc. 1976, 98, 4677; (b)
Bowden, B. F.; Braekman, J.-C.; Coll, J. C.; Mitchell, S. Aus. J. Chem. 1980, 33, 927; (c) Ochi, M.; Watanbe, M.; Miura, I.; Taniguchi, M.; Tokoroyama, T. Chem. Lett. 1980, 1229; (d) Crews, P.; Klein, T. E.; Hogue, E. R.; Myers, B. L. J. Org. Chem. 1982, 47, 811.

3. For synthetic efforts towards dolabellane framework, see: (a) Miyaoka, H.; Isaji, Y.; Mitome, H.; Yamada, Y. Tetrahedron 2003, 59, 61; (b) Miyaoka, H.; Baba, T.; Mitome, H.; Yamada, Y. Tetrahedron Lett. 2001, 42, 9233; (c) Miyaoka, H.; Isaji, Y.; Kajiwara, Y.; Kunimune, I.; Yamada, Y. Tetrahedron Lett. 1998, 39, 6503; (d) Corey, E. J.; Kania, R. S. Tetrahedron Lett. 1998, 39, 741; (e) Kato, N.; Higo, A.; Wu, X.; Takishita, H. Heterocycles 1997, 46, 123; (f) Corey, E. J.; Kania, R. S. J. Am. Chem. Soc. 1996, 118, 1229; (g) Jenny, L.; Borshberg, H.-J. Helv. Chim. Acta 1995, 78, 715; (h) Zhu, Q.; Qiao, L.; Wu, Y.-L. J. Org. Chem. 2001, 66, 2692; (i) Zeng, Z.; Xu, X. Tetrahedron Lett. 2000, 41, 3459; (j) Zhu, Q.; Fan, K.-Y.; Ma, H.-W.; Qiao, L.-X.; Wu, Y.-L.; Wu, Y. Org. Lett. 1999, 1, 757; (k) Zhu, Q.; Qiao, L.-X.; Wu, Y.; Wu, Y.-L. J. Org. Chem. 1999, 64, 2428; (1) Luker, T.; Whitby, R. J. Tetrahedron Lett. 1996, 37, 7661; (m) Kato, N.; Higo, A.; Nakanishi, K.; Wu, X.; Takeshita, H. Chem. Lett. 1994, 1967; (n) Mehta, G.; Karra, S. R.; Krishnamurthy, N. Tetrahedron Lett. 1994, 35, 2761; (o) Williams, D. R.; Coleman, P. J.; Henry, S. S. J. Am. Chem. Soc. 1993, 115, 11654; (p) Williams, D. R.; Coleman, P. J.; Nevill, C. R.; Robinson, L. A. Tetrahedron Lett. 1993, 34, 7895.

4. For synthetic studies towards dolastane diterpenoids, see: (a) Tuckett, M. W.; Watkins, W. J.; Whitby, R. J. Tetrahedron Lett. 1998, 39, 123; (b) Williams, D. R.; 
Coleman, P. J. Tetrahedron Lett. 1995, 36, 39; (c) Kato, N.; Higo, A.; Nakanishi, K.; Wu, X.; Takeshita, H. Chem. Lett. 1994, 1967; (d) Piers, E.; Friesen, R. W. Can. J. Chem. 1992, 70, 1204; (e) Mehta, G.; Krishnamurthy, K. J. Am. Chem. Soc. 1991, 113, 5765; (f) Majetich, G.; Song, J. S.; Ringold, C.; Nemeth, G. A.; Newton, M. G. Tetrahedron Lett. 1990, 31, 2239; (g) Majetich, G.; Song, J. S.; Ringold, C.; Nemeth, G. A. Tetrahedron Lett. 1990, 31, 2239; (h) Matsuo, A.; Yoshida, Kenichiro; N. M. J. Chem. Res. Synop. 1990, 22; (i) Piers, E.; Friesen, R. W. J. Chem. Soc., Chem. Commun. 1988, 125; (j) Mehta, G.; Krishnamurthy, N. Tetrahedron Lett. 1987, 28, 5945; (k) Seto, H.; Fujimoto, Y.; Tatsuno, T.; Yoshioka, H. Synth. Commun. 1985, 15, 1217; (1) Pattenden, G.; Robertson, G. M. Tetrahedron Lett. 1986, 27, 399; (m) Piers, E.; Friesen, R. W. J. Org. Chem. 1986, 51, 3405; (n) Paquette, L. A.; Lij, Ho-S.; Belmont, D. T.; Springer, J. P. J. Org. Chem. 1986, 51, 4807; (o) Rigby, J. H.; Wilson, J. Z.; Senanayake, C. Tetrahedron Lett. 1986, 27, 3329; (p) Begley, M. J.; Pattenden, G.; Robertson, G. M. J. Chem. Soc., Perkin Trans. 1 1988, 1085; (q) McConnell, O. J.; Fenical, W.; Hirotsu, K.; Clardy, J. Tetrahedron 1981, 37, 1237; (r) Belmont, D. T.; Paquette, L. A. J. Org. Chem. 1985, 50, 4102.

5. (a) Bowden, B. F.; Coll, J. C.; Gulbis, J. M.; Mackay, M. F.; Willis, R. H. Aus. J. Chem. 1986, 39, 803; (b) Kobayashi, M.; Son, B.-W.; Fujiwara, T.; Kyogoku, Y.; Kitagawa, I. Tetrahedron Lett. 1984, 25, 5543; (c) Bowden, B. F.; Braekman, J.-C.; Coll, J. C.; Mitchell, S. J. Aus. J. Chem. 1980, 33, 927; (d) Bowden, B. F.; Coll, J. C.; Mitchell, S. J.; Stokie, G. J.; Blount, J. F. Aus. J. Chem. 1978, 31, 2039.

6. (a) Brady, S. F.; Singh, M. P.; Janso, J. E.; Clardy, J. J. Am. Chem. Soc. 2000, 122, 2116; (b) Brady, S. F.; Bondi, S. M.; Clardy, J. J. Am. Chem. Soc. 2001, 123, 9900; (c) Singh, M. P.; Janso, J. E.; Luckman, S. W.; Brady, S. F.; Clardy, J.; Greenstein, M.; Maiese, W. M. J. Antibiot. 2000, 53, 256

7. (a) Mehta, G.; Umarye, J. D. Org. Lett. 2002, 4, 1063; (b) Mehta, G.; Umarye, J. D.; Gagliardini, V. Tetrahedron Lett. 2002, 43, 6975; (c) Mehta, G.; Umarye, J. D.; Srinivas, K. Tetrahedron Lett. 2003, 44, 4233; (d) Snider, B. B.; Hawryluk, N. A. Org. Lett. 2001, 3, 569; (e) Dudley, G. B.; Danishefsky, S. Org. Lett. 2001, 3, 2399; (f) Dudley, G. B.; Tan, D. S.; Kim, G.; Tanski, J. M.; Danishefsky, S. J. Tetrahedron Lett. 2001, 42, 6789; (g) Magnus, P.; Waring, M. J.; Ollivier, C.; Lynch, V. Tetrahedron Lett. 2001, 42, 4947; (h) Snider, B. B.; Shi, B. Tetrahedron Lett. 2001, 42, 9123; (i) Shipe, W. D.; Sorensen, E. J. Org. Lett. 2002, 4, 2063; (j) Nguyen, T. M.; Lee, D. Tetrahedron Lett. 2002, 43, 4033; (k) Boyer, F.-D.; Hanna, I. Tetrahedron Lett. 2002, 43, 7469; (1) Nakazaki, A.; Sharma, U.; Tius, M. A. Org. Lett. 2002, 4, 3363; (m) Nguyen, T. M.; Seifert, R. J.; Mowrey, D. R.; Lee, D. Org. Lett. 2002, 4, 3959; (n) Magnus, P.; Ollivier, C. Tetrahedron Lett. 2002, 43, 9605; (o) Tan, D. S.; Dudley, G. B.; Danishefsky, S. J. Angew. Chem., Int. Ed. 2002, 41, 2185; (p) Lin, S.; Dudley, G. B.; Tan, D. S.; Danishefsky, S. J. Angew. Chem., Int. Ed. 2002, 41, 2188; (q) Shi, B.; Hawryluk, N. A.; Snider, B. B. J. Org. Chem. 2003, 68, 1030.

8. (a) Williams, D. R., Jr.; Heidebrecht, R. W. J. Am. Chem. Soc. 2003, 125, 1843; (b) William, D. R.; Coleman, P. J. Tetrahedron Lett. 1995, 36, 35.
9. All new compounds reported here are racemic and were duly characterized on the basis of spectral (IR, ${ }^{1} \mathrm{H}$ and ${ }^{13} \mathrm{C}$ NMR) and analytical data. Selected spectral data: compound 10a: ${ }^{1} \mathrm{H}$ NMR $\left(300 \mathrm{MHz}, \mathrm{CDCl}_{3}\right) \delta 5.66(\mathrm{~d}$, $J=1.5 \mathrm{~Hz}, 1 \mathrm{H}), 5.64-5.61(\mathrm{~m}, 1 \mathrm{H}), 5.34(\mathrm{~d}, J=10.8 \mathrm{~Hz}$, $1 \mathrm{H}), 4.86(\mathrm{dd}, J=7.8,1.5 \mathrm{~Hz}, 1 \mathrm{H}), 4.38(\mathrm{dd}, J=7.8,5.4$ $\mathrm{Hz}, 1 \mathrm{H}), 3.51(\mathrm{ABq}, J=10.8 \mathrm{~Hz}, 2 \mathrm{H}), 2.54(\mathrm{dt}, J=14.1$, $3.3 \mathrm{~Hz}, 1 \mathrm{H}), 2.31-2.03$ (series of $\mathrm{m}, 4 \mathrm{H}), 1.82-1.68(\mathrm{~m}$, $2 \mathrm{H}), 1.48(\mathrm{~s}, 3 \mathrm{H}), 1.38-1.31(\mathrm{~m}, 2 \mathrm{H}), 1.36(\mathrm{~s}, 3 \mathrm{H}), 1.12(\mathrm{~d}$, $J=7.2 \mathrm{~Hz}, 3 \mathrm{H}), 1.06(\mathrm{~d}, J=6.6 \mathrm{~Hz}, 3 \mathrm{H}), 1.05(\mathrm{~s}, 3 \mathrm{H})$, $0.99(\mathrm{~d}, J=6.6 \mathrm{~Hz}, 3 \mathrm{H}) ;{ }^{13} \mathrm{C} \mathrm{NMR}\left(75 \mathrm{MHz}, \mathrm{CDCl}_{3}\right) \delta$ $154.4,130.2,124.9,123.1,112.7,83.0,79.8,72.9,65.5$, $62.2,50.2,46.4,39.8,36.7,34.3,30.1,28.3,27.9,26.3$, 23.3, 22.6, 18.3, 13.6; HRMS: calcd for $\mathrm{C}_{23} \mathrm{H}_{36} \mathrm{O}_{4} \mathrm{Na}$ $\left(\mathrm{M}^{+}+\mathrm{Na}\right)$ : 399.2511; Found: 399.2491; compound 13: ${ }^{1} \mathrm{H}$ NMR (300 MHz, CDCl $\left.{ }_{3}\right){ }^{1} \mathrm{H} \mathrm{NMR}\left(300 \mathrm{MHz}, \mathrm{CDCl}_{3}\right)$ $\delta 6.60(\mathrm{~s}, 1 \mathrm{H}), 6.53-6.51(\mathrm{~m}, 2 \mathrm{H}), 5.93(\mathrm{~d}, J=3 \mathrm{~Hz}, 1 \mathrm{H})$, $5.00(\mathrm{~d}, J=6.6 \mathrm{~Hz}, 1 \mathrm{H}), 4.26(\mathrm{dd}$ as t $J=6.9 \mathrm{~Hz}, 1 \mathrm{H})$, $3.09(\mathrm{~d}, J=11.4 \mathrm{~Hz}, 1 \mathrm{H}), 2.60(\mathrm{t}, J=12.3 \mathrm{~Hz}, 1 \mathrm{H}), 2.33$ $(\mathrm{dd}, J=12.3,7.2 \mathrm{~Hz}, 1 \mathrm{H}), 2.06(\mathrm{dd}, J=14.7,6.9 \mathrm{~Hz}, 1 \mathrm{H})$, $1.93(\mathrm{~s}, 3 \mathrm{H}), 1.81-1.75(\mathrm{~m}, 1 \mathrm{H}), 1.41(\mathrm{~s}, 3 \mathrm{H}), 1.40$ (merged signal, $1 \mathrm{H}), 1.38(\mathrm{~s}, 3 \mathrm{H}), 1.31-1.25(\mathrm{~m}, 1 \mathrm{H}), 1.23$ (s, 3H), 1.09 (d, $J=6.6 \mathrm{~Hz}, 3 \mathrm{H}), 1.02$ (d, $J=6.6 \mathrm{~Hz}, 3 \mathrm{H})$, $0.88-0.83(\mathrm{~m}, 1 \mathrm{H}) ;{ }^{13} \mathrm{C}$ NMR $\left(75 \mathrm{MHz}, \mathrm{CDCl}_{3}\right) \delta 147.1$, 136.7, 133.6, 129.1, 128.9, 125.6, 120.9, 118.2, 111.8, 82.9, $79.9,62.3,49.6,38.6,37.5,32.4,28.2,26.6,26.4,25.4$, 21.2 (2C), 17.4; HRMS: calcd for $\mathrm{C}_{23} \mathrm{H}_{32} \mathrm{O}_{2} \mathrm{Na}\left(\mathrm{M}^{+}+\mathrm{Na}\right)$ : 363.2300; Found: 363.2329; compound 17: ${ }^{1} \mathrm{H}$ NMR (300 $\left.\mathrm{MHz}, \mathrm{CDCl}_{3}\right) \delta 6.12(\mathrm{dd}, J=18.0,10.5 \mathrm{~Hz}, 1 \mathrm{H}), 5.41$ (d, $J=1.8 \mathrm{~Hz}, 1 \mathrm{H}), 5.16($ br s, $1 \mathrm{H}), 5.08-4.96$ (m, 3H), 4.80 $(\mathrm{dd}, J=6.9,1.8 \mathrm{~Hz}, 1 \mathrm{H}), 4.37(\mathrm{dd}$ as t, $J=7.2 \mathrm{~Hz}, 1 \mathrm{H})$, $2.16(\mathrm{dd}$ as t, $J=13.2 \mathrm{~Hz}, 1 \mathrm{H}), 1.95$ (dd as t, $J=13.2 \mathrm{~Hz}$, $1 \mathrm{H}), 1.83(\mathrm{~s}, 3 \mathrm{H}), 1.77-1.66(\mathrm{~m}, 2 \mathrm{H}), 1.57-1.52(\mathrm{~m}, 2 \mathrm{H})$, $1.48(\mathrm{~s}, 3 \mathrm{H}), 1.34(\mathrm{~s}, 3 \mathrm{H}), 1.18(\mathrm{~s}, 3 \mathrm{H}), 1.06(\mathrm{~d}, J=6.3 \mathrm{~Hz}$, $3 \mathrm{H}), 1.01(\mathrm{~d}, J=6.3 \mathrm{~Hz}, 3 \mathrm{H}), 0.99(\mathrm{~s}, 3 \mathrm{H}) ;{ }^{13} \mathrm{C}$ NMR $(75$ $\left.\mathrm{MHz}, \mathrm{CDCl}_{3}\right) \delta 147.4,147.3$ (2C), 131.5, 115.1, 111.9, $110.6,82.7,82.4,81.5,63.2,49.4,45.5,35.8,30.1,28.2$, 27.3, 26.2, 24.1, 22.2, 21.9, 21.6, 20.0; HRMS: calcd for $\mathrm{C}_{23} \mathrm{H}_{36} \mathrm{O}_{3} \mathrm{Na}\left(\mathrm{M}^{+}+\mathrm{Na}\right)$ : 383.2562; Found: 383.2572; compound 18: ${ }^{1} \mathrm{H} \mathrm{NMR}\left(300 \mathrm{MHz}, \mathrm{CDCl}_{3}\right) \delta 6.32(\mathrm{~s}, 1 \mathrm{H})$, 5.09-5.05 (m, 1H), $4.68(\mathrm{~d}, J=6.3 \mathrm{~Hz}, 1 \mathrm{H}), 4.46$ (dd as t, $J=6.9 \mathrm{~Hz}, 1 \mathrm{H}), 2.46-2.41(\mathrm{~m}, 1 \mathrm{H}), 2.14-1.96(\mathrm{~m}, 6 \mathrm{H})$, 1.91-1.62 (m, 3H), $1.65(\mathrm{~s}, 3 \mathrm{H}), 1.56(\mathrm{~s}, 3 \mathrm{H}), 1.37(\mathrm{~s}, 3 \mathrm{H})$, $1.20-1.17(\mathrm{~m}, 1 \mathrm{H}), 1.19(\mathrm{~s}, 3 \mathrm{H}), 1.03(\mathrm{~d}, J=7.2 \mathrm{~Hz}, 3 \mathrm{H})$, $1.02(\mathrm{~d}, J=7.2 \mathrm{~Hz}, 3 \mathrm{H}), 0.98(\mathrm{~d}, J=6.9 \mathrm{~Hz}, 3 \mathrm{H}) ;{ }^{13} \mathrm{C}$ NMR $\left(75 \mathrm{MHz}, \mathrm{CDCl}_{3}\right) \delta 205.2,156.8,136.6,128.6$, 126.9, 111.5, 80.7, 51.6, 46.6, 33.8, 27.7, 27.3, 26.3, 26.1, 25.6 (br), 24.3, 23.9 (br), 17.4 (br); HRMS: calcd for $\mathrm{C}_{23} \mathrm{H}_{36} \mathrm{O}_{3} \mathrm{Na}\left(\mathrm{M}^{+}+\mathrm{Na}\right)$ : 383.2562; Found: 383.2570; compound 19: ${ }^{1} \mathrm{H}$ NMR $\left(300 \mathrm{MHz}, \mathrm{CDCl}_{3}\right) \delta 6.47(\mathrm{~s}, 1 \mathrm{H})$, $5.09(\mathrm{dd}, J=11.1,6.0 \mathrm{~Hz}, 1 \mathrm{H}), 4.76(\mathrm{~d}, J=6.3 \mathrm{~Hz}, 1 \mathrm{H})$, $4.46(\mathrm{dd}$ as t, $J=6.9 \mathrm{~Hz}, 1 \mathrm{H}), 2.91-2.84(\mathrm{~m}, 1 \mathrm{H}), 2.24$ $2.03(\mathrm{~m}, 4 \mathrm{H}), 1.89-1.68(\mathrm{~m}, 4 \mathrm{H}), 1.62(\mathrm{~s}, 3 \mathrm{H}), 1.52(\mathrm{~s}$, $3 \mathrm{H}), 1.39-1.33(\mathrm{~m}, 1 \mathrm{H}), 1.38(\mathrm{~s}, 3 \mathrm{H}), 1.20(\mathrm{~s}, 3 \mathrm{H}), 1.11-$ $1.05(\mathrm{~m}, 1 \mathrm{H}), 1.06(\mathrm{~d}, J=6.3 \mathrm{~Hz}, 6 \mathrm{H}), 1.01(\mathrm{~d}, J=6.6 \mathrm{~Hz}$, $3 \mathrm{H}) ;{ }^{13} \mathrm{C}$ NMR $\left(75 \mathrm{MHz}, \mathrm{CDCl}_{3}\right) \delta 207.8,159.1,135.6$, 128.6, 124.3, 111.9, 82.4, 81.9, 55.0, 52.4, 46.9, 33.8, 32.7, 28.2, 27.3, 26.6, 26.15, 25.2, 23.9, 23.7, 23.6, 21.6, 18.9; HRMS: calcd for $\mathrm{C}_{23} \mathrm{H}_{36} \mathrm{O}_{3} \mathrm{Na}\left(\mathrm{M}^{+}+\mathrm{Na}\right)$ : 383.2562; Found: 383.2585 ; compound 20: ${ }^{1} \mathrm{H}$ NMR $(300 \mathrm{MHz}$, $\left.\mathrm{CDCl}_{3}\right) \delta 7.30(\mathrm{~d}, J=16.5 \mathrm{~Hz}, 1 \mathrm{H}), 5.78(\mathrm{~d}, J=16.5 \mathrm{~Hz}$, $1 \mathrm{H}), 5.43$ (d, $J=1.5 \mathrm{~Hz}, 1 \mathrm{H}), 5.13$ (br s, 1H), 5.02 (br s, $1 \mathrm{H}), 4.82(\mathrm{dd}, J=6.9,1.8 \mathrm{~Hz}, 1 \mathrm{H}), 4.39(\mathrm{dd}$ as t, $J=6.9$ 
$\mathrm{Hz}, 1 \mathrm{H}), 4.18$ (q, $J=6.9 \mathrm{~Hz}, 2 \mathrm{H}), 2.14$ (dd as t, $J=13.2$ $\mathrm{Hz}, 1 \mathrm{H}), 1.96(\mathrm{dd}$ as t, $J=13.2 \mathrm{~Hz}, 1 \mathrm{H}), 1.83-1.69(\mathrm{~m}$, $2 \mathrm{H}), 1.77$ (s, 3H), 1.59-1.52 (m, 2H), $1.48(\mathrm{~s}, 3 \mathrm{H}), 1.34(\mathrm{~s}$, $3 \mathrm{H}), 1.28(\mathrm{t}, J=6.9 \mathrm{~Hz}, 3 \mathrm{H}), 1.23(\mathrm{~s}, 3 \mathrm{H}), 1.07$ (d, $J=6.6$ $\mathrm{Hz}, 3 \mathrm{H}), 1.01(\mathrm{~d}, J=6.6 \mathrm{~Hz}, 3 \mathrm{H}), 0.98(\mathrm{~s}, 3 \mathrm{H}) ;{ }^{13} \mathrm{C}$ NMR $\left(75 \mathrm{MHz}, \mathrm{CDCl}_{3}\right) \delta 167.2,157.6,146.8,146.7,131.1$, $117.1,115.7,112.0,82.6,82.3,81.4,63.2,60.2,49.6,45.8$, $35.7,30.0,28.1,27.3,26.1,24.1,22.1,21.7,21.4,19.8$, 14.3; HRMS: calcd for $\mathrm{C}_{26} \mathrm{H}_{40} \mathrm{O}_{5} \mathrm{Na}\left(\mathrm{M}^{+}+\mathrm{Na}\right)$ : 455.2773; Found: 455.2774; compound 21: ${ }^{1} \mathrm{H}$ NMR $(300 \mathrm{MHz}$, $\left.\mathrm{CDCl}_{3}\right) \delta 6.24(\mathrm{~s}, 1 \mathrm{H}), 5.74(\mathrm{~d}, J=10.8 \mathrm{~Hz}, 1 \mathrm{H}), 4.67(\mathrm{~d}$, $J=6.3 \mathrm{~Hz}, 1 \mathrm{H}), 4.46(\mathrm{t}, J=6.6 \mathrm{~Hz}, 1 \mathrm{H}), 4.11$ (q, $J=6.9$ $\mathrm{Hz}, 2 \mathrm{H}), 3.27-3.20(\mathrm{~m}, 1 \mathrm{H}), 2.50-2.30$ (m, 2H), 2.00-1.96 (m, 1H), 1.88-1.77 (m, 3H), $1.74(\mathrm{~d}, J=9.0 \mathrm{~Hz}, 3 \mathrm{H}), 1.57$ $(\mathrm{s}, 3 \mathrm{H}), 1.37(\mathrm{~s}, 3 \mathrm{H}), 1.26-1.22(\mathrm{~m}, 8 \mathrm{H}), 1.10-1.06(\mathrm{~m}$,
$1 \mathrm{H}), 1.03(\mathrm{~d}, J=6.6 \mathrm{~Hz}, 3 \mathrm{H}), 1.03(\mathrm{~s}, 3 \mathrm{H}), 1.02$ (d, $J=6.6$ $\mathrm{Hz}, 3 \mathrm{H}) ;{ }^{13} \mathrm{C} \mathrm{NMR}\left(75 \mathrm{MHz}, \mathrm{CDCl}_{3}\right) \delta 205.5,173.9$, 157.2, 138.1, 128.8, 125.3, 111.5, 60.5, 51.2 (br), 45.6, 45.5, 37.6, 29.7, 27.5 (br), 26.2, 25.4 (br), 24.0 (br), 20.7 (br), 19.0, 14.2; HRMS: calcd for $\mathrm{C}_{26} \mathrm{H}_{40} \mathrm{O}_{5} \mathrm{Na}\left(\mathrm{M}^{+}+\mathrm{Na}\right)$ : 455.2773; Found: 455.2792.

10. Spiniferin sesquiterpenes having bicyclo[4.4.1 $\left.{ }^{1,6}\right]$ undecane skeleton incorporate a bridged cycloheptatriene moiety. Isolation: (a) Cimino, G.; De Stefano, S.; Minale, L.; Trivellone, E. Tetrahedron Lett. 1975, 26, 3727; (b) Cimino, G.; De Stefano, S.; Minale, L.; Trivellone, E. Experientia 1978, 34, 1425. Synthesis: Marshall, J. A.; Conrow, R. E. J. Am. Chem. Soc. 1980, 102, 4274.

11. (a) Paquette, L. A. Tetrahedron 1997, 53, 13971; (b) Wilson, S. R. Org. React. 1993, 43, 93. 